\title{
Article
}

\section{Engineered spatial inversion symmetry breaking in an oxide heterostructure built from isosymmetric room- temperature magnetically ordered components}

\begin{abstract}
Alaria, J., Borisov, P., Dyer, M. S., Manning, T. D., Lepadatu, Serban, Cain, M. G., Mishina, E. D., Sherstyuk, N. E., Ilyin, N. A., Hadermann, J., Lederman, D., Claridge, J. B. and Rosseinsky, M. J.

Available at http://clok.uclan.ac.uk/11793/

Alaria, J., Borisov, P., Dyer, M. S., Manning, T. D., Lepadatu, Serban ORCID: 0000-0001-6221-9727, Cain, M. G., Mishina, E. D., Sherstyuk, N. E., Ilyin, N. A. et al (2014) Engineered spatial inversion symmetry breaking in an oxide heterostructure built from isosymmetric room-temperature magnetically ordered components. Chemical Science, 5 (4). p. 1599. ISSN 2041-6520
\end{abstract}

It is advisable to refer to the publisher's version if you intend to cite from the work. http://dx.doi.org/10.1039/c3sc53248h

For more information about UCLan's research in this area go to http://www.uclan.ac.uk/researchgroups/ and search for <name of research Group>.

For information about Research generally at UCLan please go to http://www.uclan.ac.uk/research/

All outputs in CLoK are protected by Intellectual Property Rights law, including Copyright law. Copyright, IPR and Moral Rights for the works on this site are retained by the individual authors and/or other copyright owners. Terms and conditions for use of this material are defined in the policies page. 
Cite this: Chem. Sci., 2014, 5, 1599

\title{
Engineered spatial inversion symmetry breaking in an oxide heterostructure built from isosymmetric room-temperature magnetically ordered components $\uparrow$
}

\author{
J. Alaria, ${ }^{\text {ab }}$ P. Borisov, ${ }^{\text {af }}$ M. S. Dyer, ${ }^{\text {a }}$ T. D. Manning, ${ }^{\text {a }}$ S. Lepadatu, ${ }^{c}$ M. G. Cain, ${ }^{c}$ \\ E. D. Mishina, ${ }^{d}$ N. E. Sherstyuk, ${ }^{d}$ N. A. Ilyin, ${ }^{d}{ }^{J}$. Hadermann, ${ }^{e}$ D. Lederman, ${ }^{f}$ \\ J. B. Claridge*a and M. J. Rosseinsky ${ }^{\star a}$
}

Received 26th November 2013 Accepted 20th January 2014

DOI: $10.1039 / \mathrm{c} 3 s c 53248 \mathrm{~h}$

www.rsc.org/chemicalscience
The oxide heterostructure $\left[\left(\mathrm{YFeO}_{3}\right)_{5}\left(\mathrm{LaFeO}_{3}\right)_{5}\right]_{40}$, which is magnetically ordered and piezoelectric at room temperature, has been constructed from two weak ferromagnetic $\mathrm{AFeO}_{3}$ perovskites with different $\mathrm{A}$ cations using RHEED-monitored pulsed laser deposition. The polarisation arises through the removal of inversion centres present within the individual $\mathrm{AFeO}_{3}$ components. This symmetry reduction is a result of combining ordering on the A site, imposed by the periodicity of the grown structure, with appropriate orientations of the octahedral tilting characteristic of the perovskite units themselves, according to simple symmetry-controlled rules. The polarisation is robust against A site interdiffusion between the two layers which produces a sinusoidally modulated occupancy that retains the coupling of translational and point symmetries required to produce a polar structure. Magnetization and magneto-optical Kerr rotation measurements show that the heterostructure's magnetic structure is similar to that of the individual components. Evidence of the polarity was obtained from second harmonic generation and piezoelectric force microscopy measurements. Modeling of the piezoresponse allows extraction of $d_{33}$ (approximately $10 \mathrm{pC} \mathrm{N}^{-1}$ ) of the heterostructure, which is in agreement with DFT calculations.

\section{Introduction}

The breaking of inversion symmetry to generate a polarization is a prerequisite for the technologically important properties of ferroand piezoelectricity used in capacitors and actuators, while the breaking of time-reversal symmetry is required for the magnetically ordered states such as antiferro- and ferromagnetism used in information storage. It is however chemically challenging to combine both properties in a single material, because there is a competition in the electronic structure requirements between

${ }^{a}$ Department of Chemistry, University of Liverpool, Liverpool, L69 7ZD, UK. E-mail: rossein@liv.ac.uk; claridge@liv.ac.uk

${ }^{b}$ Stephenson Institute for Renewable Energy, Department of Physics, University of Liverpool, Liverpool, L69 7ZE, UK

${ }^{c}$ National Physical Laboratory, Hampton Road, Teddington, Middlesex TW11 OLW, UK ${ }^{d}$ Moscow State Technical University of Radioengineering, Electronics and Automation, Vernadsky Avenue 78, 119454 Moscow, Russia

${ }^{e} E M A T$, University of Antwerp, Groenenborgerlaan 171, B-2020, Antwerp, Belgium ${ }^{f}$ Department of Physics and Astronomy, West Virginia University, Morgantown, West Virginia, 26506-6315, USA

$\dagger$ Electronic supplementary information (ESI) available: Further details on computational methods, substrate preparation, thin film growth, structural characterization, magnetic measurement, nonlinear optical measurements, electrical characterization and piezoelectric force microscopy. See DOI: $10.1039 / \mathrm{c} 3 \mathrm{sc} 53248 \mathrm{~h}$ many of the mechanisms responsible for forming each state $^{1}$ e.g. the crystal chemistry of the closed-shell $\mathrm{d}^{0} \mathrm{Ti}^{4+}$ and $\mathrm{s}^{2} \mathrm{~Pb}^{2+}$ cations driving ferroelectricity in $\mathrm{PbZr}_{1-x} \mathrm{Ti}_{x} \mathrm{O}_{3}$ does not afford the unpaired electrons required for magnetic order. It is possible to approach this problem by combining the two chemistries required, as in $\mathrm{BiFeO}_{3}$ where $\mathrm{Fe}^{3+}$ provides antiferromagnetism and $\mathrm{Bi}^{3+}$ ferroelectricity, by making composites over a range of length scales between compounds which individually display one of the two required ground states, ${ }^{2}$ or by coupling reduced spatial symmetry with the onset of magnetic order..$^{3-5}$

The $\mathrm{ABO}_{3}$ perovskite structure supports both magnetic and polar ground states. The diverse array of physical properties can be controlled via tilting of the $\mathrm{BO}_{6}$ octahedra, ${ }^{6,7}$ through the $\mathrm{B}-\mathrm{O}-\mathrm{B}$ overlap, and by ordering of cations on both the $\mathrm{A}^{8,9}$ and $\mathrm{B}$ sites. ${ }^{\mathbf{1 0}}$ Recent theoretical work ${ }^{\mathbf{1 1 - 1 4}}$ has proposed that specific combinations of cation order and tilting, originally elucidated for HRTEM defect analysis of bulk materials, ${ }^{15}$ can impose polarity on $\left(\mathrm{ABO}_{3}: \mathrm{A}^{\prime} \mathrm{BO}_{3}\right) 1: 1$ heterostructures where both components adopt the Pnma structure. This involves out-ofphase octahedral tilting along two pseudocubic $a_{\mathrm{p}}$ perovskite subcell directions denoted $a^{-}$and in-phase tilting along the third, denoted $b^{+}$, which becomes the $b$ axis of the $a_{P n m a}=\sqrt{ } 2 a_{\mathrm{p}}$, $b_{\text {Pnma }}=2 a_{\mathrm{p}}$ and $c_{\text {Pnma }}=\sqrt{ } 2 a_{\mathrm{p}}$ unit cell (Fig. 1). These design rules to produce new Hybrid Improper Ferroelectric (HIF) 

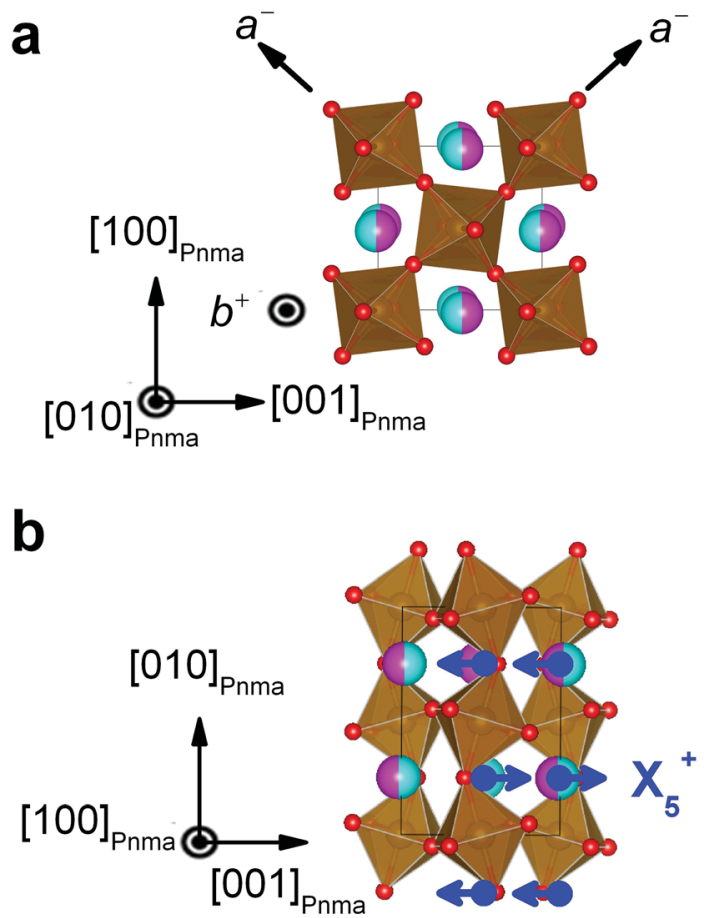

Fig. 1 Projection of the Pnma $\mathrm{GdFeO}_{3}$ structure (a) projected along the $b$ axis showing the orientation of the tilt axes and (b), projected along a highlighting the $\mathrm{X}_{5}{ }^{+} \mathrm{A}$ site antiferrodistortive displacement mode symmetry. Atoms are colored La (cyan), Y (magenta), Fe (brown) and $\mathrm{O}$ (red) throughout the manuscript.

compounds experimentally are difficult to achieve in bulk materials due to the size requirement for the A cation ordering and has been observed in the double perovskite $\mathrm{NaLaMnWO}_{6} \cdot{ }^{16}$ Metal Organic Framework (MOF) also have great potential in the discovery of new multiferroic materials ${ }^{17}$ and the concept of HIF can be generalized for MOF with a perovskite structure. ${ }^{18}$

Oxide heterostructures display emergent phenomena controlled by charge, orbital and spin reconstruction at the internal interfaces within the thin films. ${ }^{19}$ Inversion and timereversal symmetries have been broken together in tricolor heterostructures employing multiple component compositions and symmetries, ${ }^{20}$ or by using substrate-induced strain. ${ }^{21}$ The mechanism described previously is distinct from compositionally generated polarity in tricolor superlattices ${ }^{20,22}$ as it operates in two-component isosymmetric systems through the coupling of point symmetry to translational compositional modulation. This mechanism by its nature will promote the coupling between polarization and magnetization. ${ }^{23}$

By increasing the separation between the polar interfaces while retaining the inversion symmetry breaking by coupling A site modulation to correctly oriented octahedral rotation, a polarity is generated in a heterostructure built from two magnetically ordered perovskites, thereby combining both broken space inversion and time-reversal symmetries at room temperature.

\section{Experimental \& computational details}

$\left(\mathrm{YFeO}_{3}\right)_{5} /\left(\mathrm{LaFeO}_{3}\right)_{5}, \quad\left(\mathrm{YFeO}_{3}\right)_{4} /\left(\mathrm{LaFeO}_{3}\right)_{4}, \quad \mathrm{YFeO}_{3}$, and $\mathrm{LaFeO}_{3}$ films with a total thickness of $160 \mathrm{~nm}$ on atomically flat (101)
$\mathrm{DyScO}_{3}$ single crystal substrates and a $160 \mathrm{~nm}\left(\mathrm{YFeO}_{3}\right)_{5} /$ $\left(\mathrm{LaFeO}_{3}\right)_{5}$ film on atomically flat (001) $\mathrm{SrTiO}_{3}$ were grown using pulsed laser deposition with a $\operatorname{KrF}(248 \mathrm{~nm})$ ultraviolet excimer laser. The $\mathrm{SrTiO}_{3}$ and $\mathrm{DyScO}_{3}$ substrates were prepared following the procedure described in the literature; ${ }^{24,25}$ the surface morphology of the treated substrate showed clear step and terraces structure (Fig. S3 $\uparrow$ ). The targets were prepared by solid state reaction of the dried oxides, the deposition was carried out under 0.7 mTorr of high purity $\mathrm{O}_{2}$ with a substrate temperature of $700{ }^{\circ} \mathrm{C}$ and a laser fluence of $1 \mathrm{~J} \mathrm{~cm}^{-2}$ and a frequency of 3-5 Hz. The growth was monitored using a differentially pumped RHEED system by recording the specular reflection intensity variation.

X-ray diffraction was carried out using a Panalytical X'Pert Pro MRD equipped with a four bounce Ge (220) monochromator.

High angle annular dark field scanning transmission electron microscopy (HAADF-STEM) images were acquired on an FEI Titan 50-80 microscope equipped with a probe aberration corrector and operated at $300 \mathrm{kV}$. The sample was prepared with focused ion beam (FIB) on a Helios NanoLab 650.

The magnetization of the films grown on $\mathrm{SrTiO}_{3}$ substrates was measured in a MPMS SQUID magnetometer (Quantum Design) at room temperature with the applied field parallel and perpendicular to the film surface.

Magnetic hysteresis loops of the films grown on $\mathrm{DyScO}_{3}$ were recorded using the magneto-optical Kerr effect (MOKE) in a setup equipped with an electromagnet capable of producing magnetic fields of up to 20 kOe in longitudinal geometry at room temperature. A $40 \mathrm{~mW} 409 \mathrm{~nm}$ laser was used with an angle of incidence of $14^{\circ}$ with respect to the substrate normal. The initial beam was linearly polarized perpendicular to the plane of incidence (S-polarization) and modulated by a photoelastic modulator at $f=50 \mathrm{kHz}$. The second harmonic component, $2 \mathrm{f}$, proportional to the angle of the Kerr-rotation, was extracted by a lock-in amplifier from the intensity of the reflected light.

Second harmonic generation was measured at room temperature using an $800 \mathrm{~nm}$ Ti-sapphire laser at a repetition rate of $82 \mathrm{MHz}$, the pulse average power and duration were 5-30 $\mathrm{mW}$ and $100 \mathrm{fs}$, respectively. The fundamental beam, whose polarization was varied with a half-wave plate, was focused onto the sample with the minimal cross-section of about $20 \mu \mathrm{m}$ at the angle of incidence of $45^{\circ}$. SHG polar dependence was measured with the analyzer fixed at a polarization parallel (P) to the plane of incidence, and with the substrate edges fixed parallel to the analyzer position (=at 45 degrees to the in-plane axis of the polar domain). The input polarization was rotated continuously by $360^{\circ}$ degrees starting from polarization parallel $(\mathrm{P})$ to the plane of incidence. A schematic of the geometry used for this experiment is presented on Fig. S12. $\dagger$

The piezoelectric properties of the samples were characterized using a modified piezoelectric force microscopy (PFM) technique. ${ }^{26}$ The samples were patterned with interdigitated electrodes using optical lithography, forming an alternating sequence of electrical ground and potential lines. The electrodes were Ti $20 \mathrm{~nm} / \mathrm{Au} 80 \mathrm{~nm}$ with a width and spacing of 
$30 \mu \mathrm{m}$ each. For the PFM-based measurements, the voltage was applied directly to the interdigitated electrodes and the lateral and vertical sample surface displacements were measured by monitoring the cantilever displacements using calibrated lockin techniques. The sensitivities of the vertical and lateral signals were calibrated using a piezo stack which was in turn calibrated using an accurate laser Doppler vibrometer. For the lateral signal, the displacements measured in this work lie in the linear region, below the onset of tip-surface sliding, as we have verified. The vertical signal also lies in the linear region with indentation of the tip into the surface being negligible. For displacement profile measurements the excitation voltage had 1 $\mathrm{V}$ amplitude at $10 \mathrm{kHz}$. The measurement frequency was chosen away from any cantilever resonance. For the scanning mode, to increase the signal to noise ratio, the imaging was done at a cantilever resonance frequency, typically around $320 \mathrm{kHz}$. In this case, the PFM imaging is restricted to measurements of the vertical displacement.

All the crystal structures presented in this work were drawn using the program VESTA. ${ }^{27}$

Superlattices were constructed in supercells based on the $\mathrm{GdFeO}_{3}$ structure $\left(\sqrt{ } 2 a_{\mathrm{p}} \times n a_{\mathrm{p}} \times \sqrt{ } 2 a_{\mathrm{p}}, a_{\mathrm{p}} \approx 3.9 \AA\right)$ 列. Spin polarized calculations on Fe containing compounds were performed with G-type antiferromagnetic ordering consistent with the parent materials. The unit cell and atomic positions were optimized until the forces acting on each atom were less than $0.001 \mathrm{eV}^{-1}$. The fully relaxed structures were symmetrized using the FINDSYM programme ${ }^{28}$ and the symmetric structures then further optimized to give a final structure. The resulting tight level of convergence was required to allow for an accurate calculation of the polarization for each structure.

Periodic density functional theory calculations were performed using the PBE functional ${ }^{29}$ with the addition of an onsite Hubbard term for Fe with $U_{\text {eff }}=4 \mathrm{eV} .{ }^{30}$ A plane-wave cutoff energy of $550 \mathrm{eV}$ was used, and a $k$-point grid of $6 \times 6 \times 4$ for the 1-1 structures (the number of $k$-points in the $c$ direction was reduced according to the height of the supercell). The geometry optimization calculations were performed using VASP ${ }^{31}$ and the projector augmented wave method. ${ }^{32}$

Following geometry optimization, the static polarization was calculated in three ways. In the simplest method, the polarization was calculated using nominal static charges on each of the ions $\left(\mathrm{Ln}^{3+}, \mathrm{Y}^{3+}, \mathrm{Fe}^{3+}, \mathrm{Ga}^{3+}, \mathrm{O}^{2-}\right)$, and calculating their displacement away from a non-polar reference structure with $\mathrm{Cmmm}$ symmetry for odd and $C 2 / \mathrm{m}$ symmetry for even structures. Electronic effects were then included by using calculated Born effective charges in place of the static nominal charges. These were calculated for the final polar structure using density functional perturbation theory as implemented in VASP. ${ }^{33}$ Finally, the polarization was also calculated for certain structures using the Berry phase method using the Quantum Espresso $\operatorname{code}^{34}$ using the VASP optimised structure and equivalent DFT settings. These three methods are described in more detail in a recent review. ${ }^{35}$ The relaxed-ion piezoelectric tensor was calculated using the implementation in VASP.

Classical force-field calculations were using the General Utility Lattice Programme (GULP). ${ }^{36}$ The force-field was constructed from a long range electrostatic part, which uses a shell model $^{37}$ to include a level of polarisability, and short-range Buckingham potentials ${ }^{38}$ acting between pairs of ions, excluding cation-cation pairs. The parameters for the potential were chosen from previous literature, ${ }^{39,40}$ and tested on bulk $\mathrm{YFeO}_{3}$ and $\mathrm{LaFeO}_{3}$. Experimental cell parameters for these orthoferrites were reproduced with a maximum error of $2 \%$. Partial occupancy of sites was treated using the mean-field approach in which the electrostatic and Buckingham potentials of that site are the average of the potentials for each species on that site, weighted by their occupancies.

\section{Results and discussion}

\section{Symmetry considerations}

Tilting cannot produce polar symmetries in single A site perovskites. ${ }^{41}$ However, in the presence of cation ordering on the A site along the $[010]_{P n m a}$ direction which is coupled to inphase tilting of the octahedra along the same direction, a spontaneous electrical polarization arises. ${ }^{\mathbf{1 1}-14}$ This is an improper ferroelectric ${ }^{\mathbf{4 2}}$ as the primary order parameter is the zone boundary octahedral tilting. The polarization arises from non-cancellation of antiferrodistortive displacements in the $\mathrm{X}_{5}{ }^{+}$mode (of the parent cubic perovskite) at the interfaces between the blocks, producing a polarization density wave as seen in the lead-free ferroelectric $\mathrm{Bi}_{0.72} \mathrm{La}_{0.28}\left(\mathrm{Fe}_{0.46} \mathrm{Ti}_{0.27} \mathrm{Mg}_{0.27}\right)$ $\mathrm{O}_{3}{ }^{43}$ This observation can be further generalized in terms of the removal of inversion symmetry within the $\mathrm{X}_{5}{ }^{+}$mode present in tilted perovskites where there are perpendicular in phase and out of phase tilts, as highlighted in Fig. 1b. Indeed for single layer A site ordering Kishida et al. ${ }^{\mathbf{1 5}}$ have determined the symmetry of all possible tilt systems and cation ordering directions. Of these by far the most common in known perovskites is the $a^{-} b^{+} a^{-}$tilt scheme producing the Pnma symmetry discussed above.

The generation of the sharp-interface A site alternation in single perovskite superlattices is challenging experimentally.

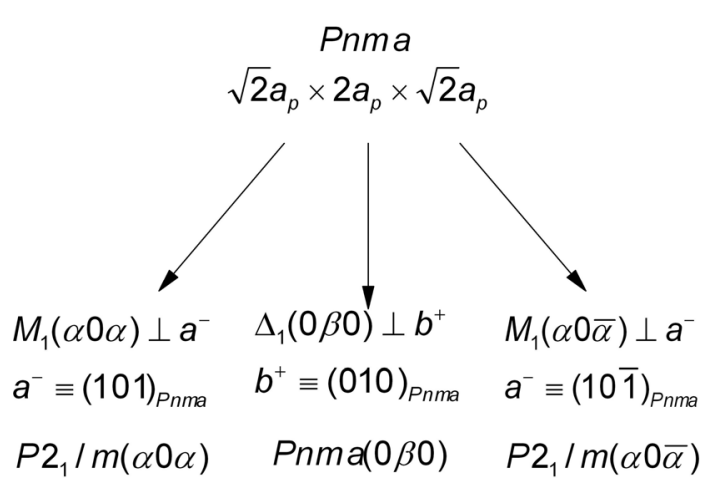

Fig. 2 Layered A cation order perpendicular to the three Pnma axes can be treated as modulations at the $M(\alpha 0 \gamma)$ point, in the Brillouin zone for Pnma, ${ }^{47}$ for the $a^{-}$axes (the general point corresponds to general planar orders perpendicular to the ac plane and the special cases $(\alpha 0 \alpha)$, $(\alpha 0-\alpha)$ to the two planes of interest) and $\Delta(0 \beta 0)$ point for the $b^{+}$axis, subscripts refer to particular irreducible representations which describe the modulation. 
We therefore used symmetry to generalize the crystal chemical rules defined previously ${ }^{11-14}$ by extending the size of the distinct $\mathrm{ABO}_{3}$ blocks and found that superlattices in which odd numbers of unit cells of both components are present retain the uncancelled displacements at the interfaces between the two components and are thus still polar in space group $P 2{ }_{1} m a$ (standard setting $P m c 2_{1}$ ) when the growth direction is parallel to the axis about which the in-phase tilting takes place. This generalization was achieved by describing the A site cation ordering perpendicular to either the $a^{-}$or $b^{+}$tilt in terms of modulated structures in superspace. ${ }^{\mathbf{4 4 - 4 6}}$ Cation ordering on the A site will increase the periodicity along one of the three pseudocubic perovskite subcell axes, according to the relative orientation of the ordering direction and the octahedral tilts. The resulting symmetries can be economically evaluated in a modulated structure description and are summarized on Fig. 2. Selection of the Pnma $b$ axis as the ordering direction produces a generalised supercell described by the $\left(0, k_{y}, 0\right)$ reciprocal space wavevector which spans the $\Delta_{1}$ irreducible representation of Pnma and affords superspace groups described as Pnma(0 $\beta 0)$. Here the modulation vector $\beta$ is the selected value of $k_{y}$ and is the inverse of the multiplication of the $2 a_{\mathrm{p}}$ repeat of the original cell along the ordering direction in real space. Orderings along the out-of-phase tilt axes are described similarly as $M_{1}(\alpha 0 \alpha)$ and $M_{1}(\alpha 0-\alpha)$ affording $P 2_{1} / m(\alpha 0 \alpha)$ and $P 2_{1} / m(\alpha 0-\alpha)$ superspace groups - here some symmetry elements of Pnma do not leave the wavevector invariant and hence the symmetry is lowered. The Brillouin zone and the definition of the $k$-vector types for the Pnma space group can be found on the Bilbao Crystallographic Server. ${ }^{47}$

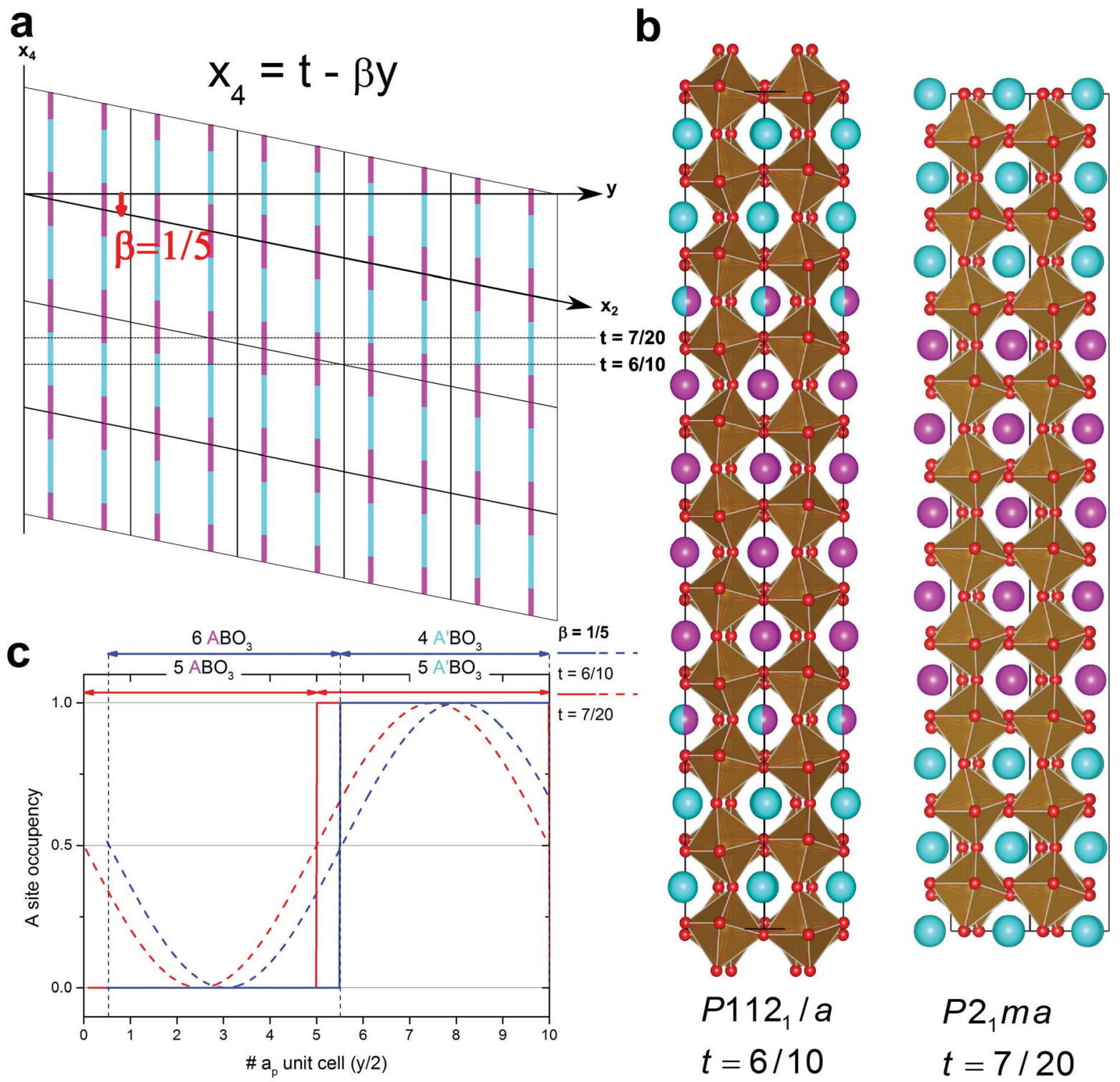

Fig. 3 (a) Projection of the $x_{4}, x_{2}$ plane for $\beta=1 / 5$ showing how the stacking sequence can be built up, here projections parallel to $y$ correspond to structures in real space, where a cyan occupation domain is intersected a La is present on the A site and where a magenta one is intersected a $Y$ is present. ( $\mathrm{b}$ and $\mathrm{c}$ ) Two example projections corresponding to origins of $t=7 / 20$ (red functions in c)and $6 / 10$ (blue functions in c) are shown. $x_{2}$ is the superspace coordinate related to $y$ as defined $i^{44}$ such that the occupancy is periodic in $x_{2}$ for unit translations along $y$. 
To describe the modulation in occupancy we define a fourth dimension $x_{4}=t-\beta y$ (Fig. 3a) in superspace, to define the atomic positions in terms of the fractional coordinates and occupancies of the Pnma subcell plus the effect of the modulation on these parameters on moving from one real space subcell to another (as the modulation has no component along $x$ or $z$, the position on $x_{4}$ is unchanged as we project cells into real space along these directions). If the structure is incommensurate the origin $t$ is arbitrary as all points on $x_{4}$ are projected. However when we consider commensurate structures, only certain points on $x_{4}$ are visited and the choice of $t$ is important for determining the real space structure. Independent of the exact nature of the modulation, the symmetry of the resulting structure can be determined from the origin $t$ and the modulation vector. ${ }^{48,49}$ Each choice of origin $t$ along $x_{4}$ corresponds to a specific real space A site occupancy and the full higher dimensional structure describes all possible combinations of A site occupancies within the periodicity defined by $\beta$.

The spatial variation of the A cation occupancy within the supercell can then be described by the periodicity of the ordering (defined by $\alpha$ and $\beta$ above) and the nature of the site occupancy variation. This is described by any periodic function of $x_{4}$ : a crenel function (Fig. 3c) of $x_{4}$ gives occupancies of zero or one at each $\mathrm{A}$ site, ${ }^{50}$ and the width of the crenel function then defines the superlattice stoichiometry, with a width of 0.5 corresponding to a $50: 50$ ratio of the two different A site cations and a width of 0.6 corresponding to a $60: 40$ ratio. Fig. 3 shows two possible origin choices for a $50: 50$ superlattice with $\beta=1 / 5$ that is polar in $P 2{ }_{1} m a$ symmetry and a mixed superlattice that is non-polar in $P 112_{1} / a$. Only these two types of origin choices are compatible with the crenel description here.

This can be generalized to describe any A site crenel-like superlattice such that for 50 : 50 lattices all $\beta=1 /(2 N)$ structures with even numbers of unit cells are centrosymmetric whilst $\beta=$ $1 /(2 N+1)$ are polar. This result, i.e. odd-odd superlattices are polar, even-even ones are not holds for non $50: 50$ lattices with odd-odd or even-even combinations (see above for the 6:4 case). Odd-even cases are described by $\beta=2 N /(2 N+1)$ and are all centrosymmetric. More complex stacking sequences are of course possible and their symmetries can be similarly deduced. A similar argument can be made for B site ordered crenels showing that for $50: 50$ lattices all $\beta=1 /(2 N+1)$ structures are centrosymmetric whilst all $\beta=1 /(2 N)$ are polar. Similar treatment of $P 2_{1} / m(\alpha 0 \alpha)$ and $P 2_{1} / m(\alpha 0-\alpha)$ superspace structures show that crenel based structures are non-polar $P 2_{1} / m$ supercells.

The above approach can also be generalized to arbitrary modulation functions e.g. for simple trigonometric functions when the origin is chosen such that the centre of the layer represents the maximum in occupation - illustrated by the sine wave modulation functions in Fig. $3 c$ - the same rules are obtained. Note that an arbitrary choice of origin for the sinusoidal modulation function will give a polar structure $(P 11 a)$, though this is induced by cation order by analogy with multiple color lattices, rather than by removal of specific symmetry elements.

\section{Materials selection and computational analysis}

Materials selection began with the choice of $\mathrm{Fe}^{3+}$ as the $\mathrm{B}$ site cation, as the strong $\mathrm{d}^{5}-\mathrm{d}^{5}$ superexchange interactions give high antiferromagnetic ordering temperatures and with $\mathrm{A}=\mathrm{Ln}^{3+}$ the resulting tilting of the octahedra due to tolerance factor considerations gives all $\mathrm{LnFeO}_{3}$ perovskites the required Pnma symmetry. The resulting non-centric $\mathrm{B}-\mathrm{O}-\mathrm{B}$ superexchange pathways permit the formation of a weak ferromagnetic moment due to Dzialoshinsky-Moriya (DM) canting. ${ }^{51}$

A consequence of the previous symmetry arguments is that for stacking of the individual $\mathrm{ABO}_{3}$ and $\mathrm{A}^{\prime} \mathrm{BO}_{3}$ components perpendicular to $b^{+}$, superlattices in which either component is present in even numbered blocks are not polar due to displacement cancellation (Fig. S1 $\dagger$ ), for stackings perpendicular to $\mathrm{a}^{-}$all superlattices are non-polar. The effect of the size of the Pnma building blocks on the total polarization was investigated computationally. These calculations showed that for odd numbered superlattices, extending the blocks expands the region between the $\mathrm{ABO}_{3}-\mathrm{A}^{\prime} \mathrm{BO}_{3}$ interfaces with almost nonpolar bulk-like layers, such that the polarization is inversely proportional to the block thickness (Fig. 4 and 5). These generalized rules allow some flexibility in achieving the

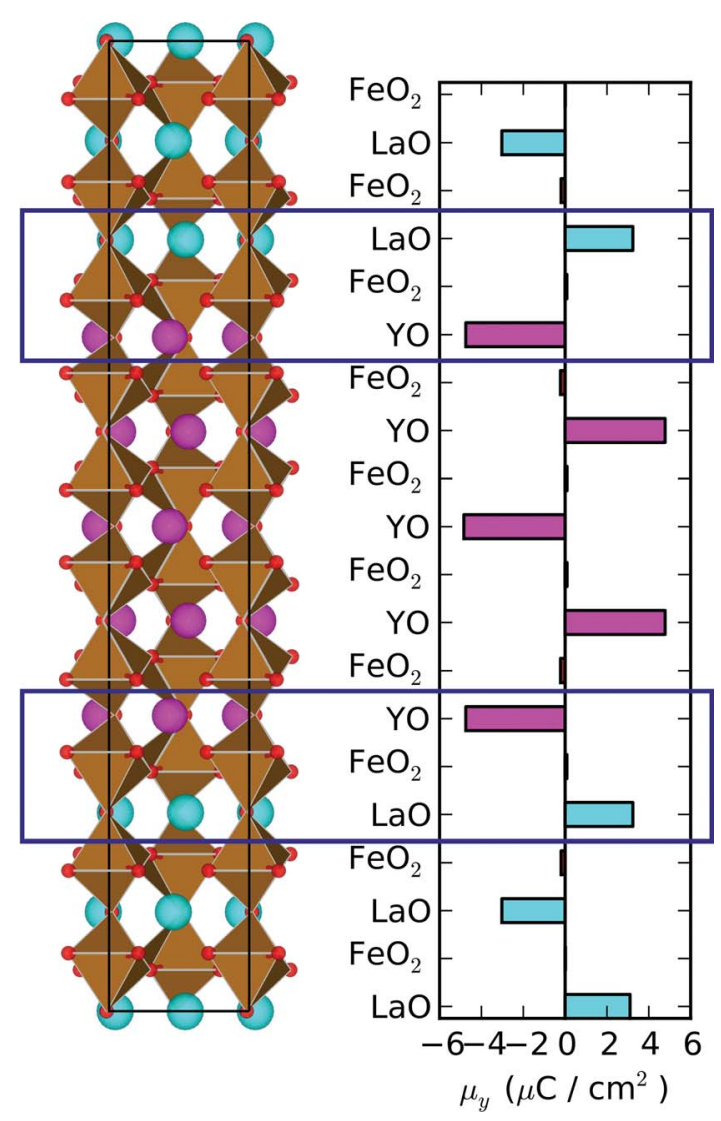

Fig. 4 The calculated structure of the $\left(\mathrm{LaFeO}_{3}\right)_{5}\left(\mathrm{YFeO}_{3}\right)_{5}$ superlattice is shown alongside the contribution from each layer to the overall polarization. The box highlights the interface region where the local centre of symmetry is lost, leading to different contributions to the polarization from the $\mathrm{AO}$ layers above and below the interface and a finite overall polarization. 


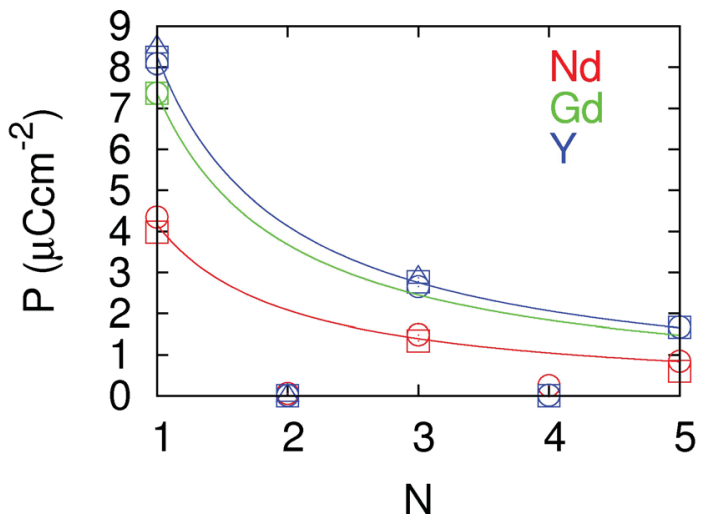

Fig. 5 Polarization $(P)$ versus the number of perovskite unit cells $(N)$ in each block for $\mathrm{LaFeO}_{3}-\mathrm{LnFeO}_{3}$ superlattices $(\mathrm{Ln}=\mathrm{Nd}, \mathrm{Gd}, \mathrm{Y})$. Data is plotted for three methods of calculating polarization: static charges (squares), Born effective charges (circles) and Berry phase (triangles). The function $P 1 / N$, where $P 1$ is the average calculated polarization for the $1-1$ superlattices, is plotted as a line for each family.

required A site compositional modulation over these longer distances in heterostructures experimentally. Suitable $\mathrm{Ln}^{3+}$ cations must be chosen to give polarity, and calculations show that for all odd numbered superlattices the polarization is maximized with the largest possible ionic size difference between the two A cations, leading to the selection $\mathrm{A}=\mathrm{La}, \mathrm{A}^{\prime}=$
$\mathrm{Y}$ (Fig. 5). This maximises the difference between the degree of displacement within $\mathrm{AO}$ and $\mathrm{A}^{\prime} \mathrm{O}$ layers. The final requirement is that the in-phase tilt axis, $b^{+}$, should be parallel to the stacking direction of the $\mathrm{LaFeO}_{3}$ and $\mathrm{YFeO}_{3}$ blocks. Calculations reveal that for $\left(\mathrm{LaFeO}_{3}\right)_{5}\left(\mathrm{YFeO}_{3}\right)$ superlattices only small energy differences separate the possible distinct tilt orientations, the $P 2_{1} m a$ structure with the $b^{+}$axis parallel to the stacking axis being less stable by only $0.06 \mathrm{eV}$ per formula unit than the $P 2_{1} / \mathrm{m}$ structure with the $b^{+}$tilt perpendicular to the stacking direction.

\section{Growth}

Quasi-epitaxial growth at $600{ }^{\circ} \mathrm{C}$ of both selected orthoferrites on cubic substrates produces three orthorhombic domains corresponding to the different orientations of the $b^{+}$tilt. ${ }^{52-54} \mathrm{We}$ observed layer-by-layer growth for $\mathrm{LaFeO}_{3}$ on $\mathrm{SrTiO}_{3}$ [100] (STO) above $600{ }^{\circ} \mathrm{C}$ (ESI Fig. S5 $\dagger$ ); below $600{ }^{\circ} \mathrm{C}$ the surface mobility is insufficient to induce crystallinity in the growing layers. We succeeded in growing $\mathrm{YFeO}_{3} / \mathrm{LaFeO}_{3}$ heterostructures on STO (ESI Fig. S6 and S7 $\dagger$ ). Higher quality heterostructures with fewer domains arise from growth on $\mathrm{DyScO}_{3}$ [101] (DSO) (Fig. S8†). This substrate is a Pnma perovskite which can be treated to obtain a pristine $\mathrm{ScO}_{2}$-terminated surface necessary for layer by layer growth ${ }^{24}$ (Fig. S3†). The substrates used here have the $b^{+}$ tilt in-plane with two domains (ESI Fig. S4†) corresponding to the two orientations of this axis along the pseudocubic subcell directions. The good structural and dimensional match

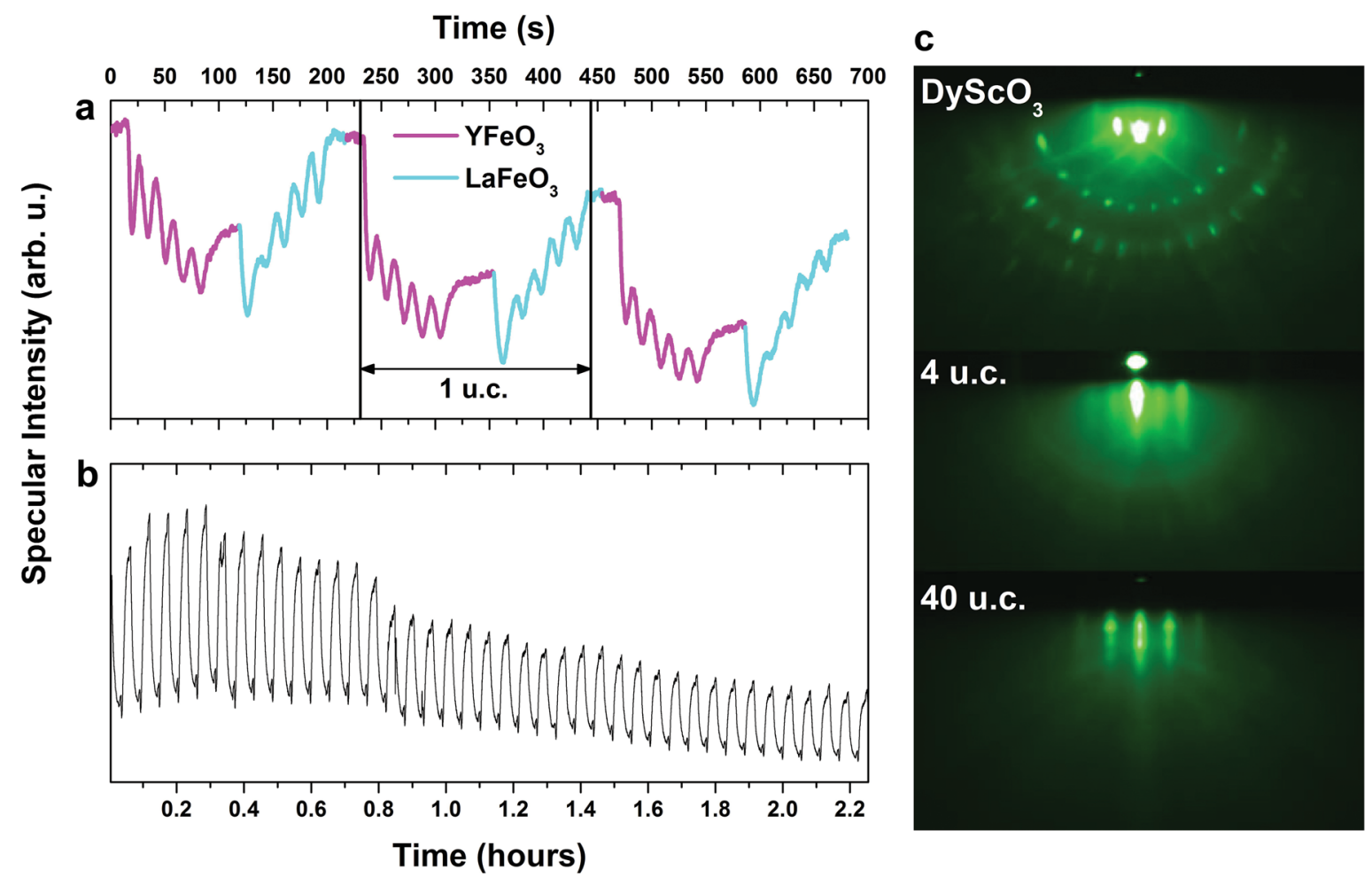

Fig. 6 (a) Specular RHEED intensity as a function of deposition time for the first 3 unit cells showing 5 clear oscillations for both $\mathrm{YFeO}_{3}$ and $\mathrm{LaFeO}_{3}$ layers. (b) Specular intensity variation for the full heterostructure showing that the layer by layer growth is sustained for the 40 unit cells with generally decreasing intensity for the $\mathrm{YFeO}_{3}$ layers and increasing intensity for the $\mathrm{LaFeO}_{3}$ layers. (c) $\mathrm{RHEED} \mathrm{pattern} \mathrm{of} \mathrm{(top)} \mathrm{ScO}_{2}$ terminated DSO substrate (middle) 4 unit cells and (bottom) 40 unit cells of the heterostructure along the [110] pseudocubic azimuth. The streaky pattern shows that the growth stays two-dimensional throughout the deposition. 
$\left(\varepsilon_{\mathrm{s}} \approx 3.5 \%\right.$ ) allows $\mathrm{YFeO}_{3}$ to grow layer-by-layer on DSO above $600{ }^{\circ} \mathrm{C}$, producing a smooth two-dimensional surface after 5 unit cells are deposited, which permits subsequent growth of $\mathrm{LaFeO}_{3}$ in layer-by-layer mode (Fig. 6). Growth was carried out at the lowest temperature which afforded the RHEED oscillations characteristic of layer-by-layer growth to minimize A cation interdiffusion between the blocks.

\section{Structural characterization}

The periodicity of the superlattice is confirmed by the presence of a low-angle reflection in the specular X-ray reflectivity corresponding to a unit cell length of $38.8 \AA$ showing that the structure is composed of 10 unit cells of the primitive perovskite (Fig. 7a). The observation of appropriately spaced satellite reflections around the fundamental $\mathrm{LnFeO}_{3}$ perovskite out of plane reflections at higher angle (Fig. 7b), confirms that the structure is composed of 5 unit cells of $\mathrm{LaFeO}_{3}$ and 5 unit cells of $\mathrm{YFeO}_{3}$. It is important to note that only first order superstructure reflections from the $10 a_{\mathrm{p}}$ cell are seen, which is consistent with a non square-wave modulation of the occupancies of the A sites between successive blocks. Interdiffusion during growth produces a sinusoidal modulation of the A site occupancy away from the centre of the blocks which will not produce harmonic diffraction features.

This is confirmed by HAADF-STEM imaging which shows some interdiffusion across the interfaces which nonetheless remain clearly present in the images (Fig. 8). The brightness in
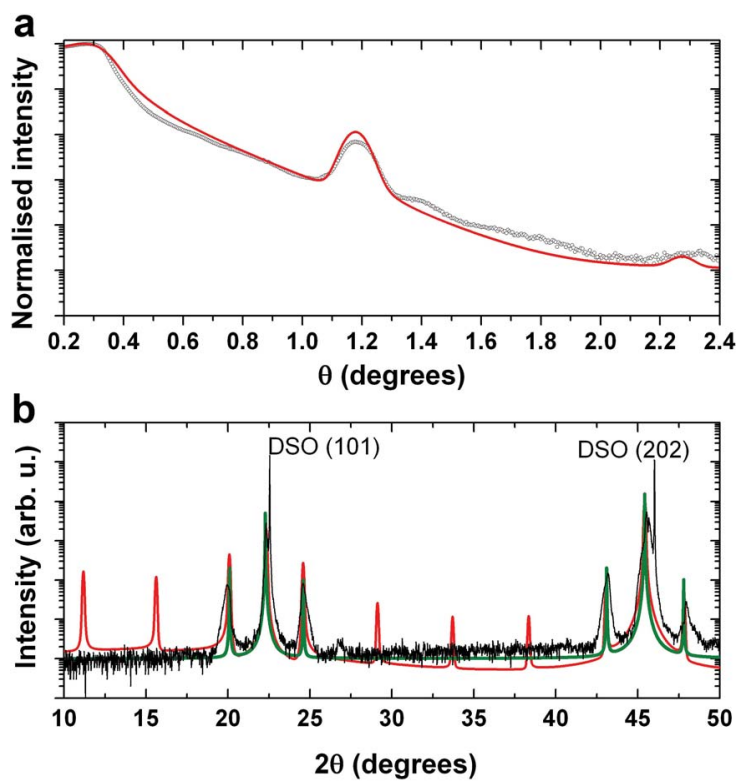

Fig. 7 (a) X-ray reflectivity of the $\left[\left(\mathrm{YFeO}_{3}\right)_{5}\left(\mathrm{LaFeO}_{3}\right)_{5}\right]_{40}$ heterostructure showing a Bragg reflection corresponding to an interatomic separation of $38.8 \AA$, the red line is a simulation of the heterostructure, the second feature around 2.3 degrees can be modeled using a surface roughness of $0.8 \mathrm{~nm}$. (b) Out of plane $\theta-2 \theta$ XRD pattern of the heterostructure, the red line corresponds to a simulation of the structure using a crenel modulation (perfect interfaces) with the expected odd harmonic reflections whereas the green line corresponds to a simulation using a sinusoidal modulation of the A cation composition. the projected columns is directly related to the average $Z$ number of the columns. The intensity profile, taken over the full width of the image, only displays maxima due to the A-cations, as those from the lighter B-cations $(Z=26)$ do not rise above the base of the A-cation peaks. The alternation between higher intensity peaks corresponding to La-rich areas $(Z=57)$ and lower intensity peaks from Y-rich areas $(Z=39)$ follows a sine wave, i.e. the transitions between La- and Y-rich areas occurs gradually consistent with the superstructure intensity variation in the X-ray diffraction.

Classical force-field calculations were carried out in order to assess the effect of the experimentally observed interlayer mixing between A-sites on polarization. To test the reliability of the force-field the force-field optimized structure of the fully ordered $\left(\mathrm{LaFeO}_{3}\right)_{5}\left(\mathrm{YFeO}_{3}\right)_{5}$ superlattice with atomically sharp interfaces was obtained, and its polarization calculated using static charges. The structure and resulting polarization of $2.5 \mu \mathrm{C} \mathrm{cm}^{-2}$ were sufficiently close to those calculated with DFT $\left(P=1.7 \mu \mathrm{C} \mathrm{cm}^{-2}\right)$ to consider the force-field as reliable. A

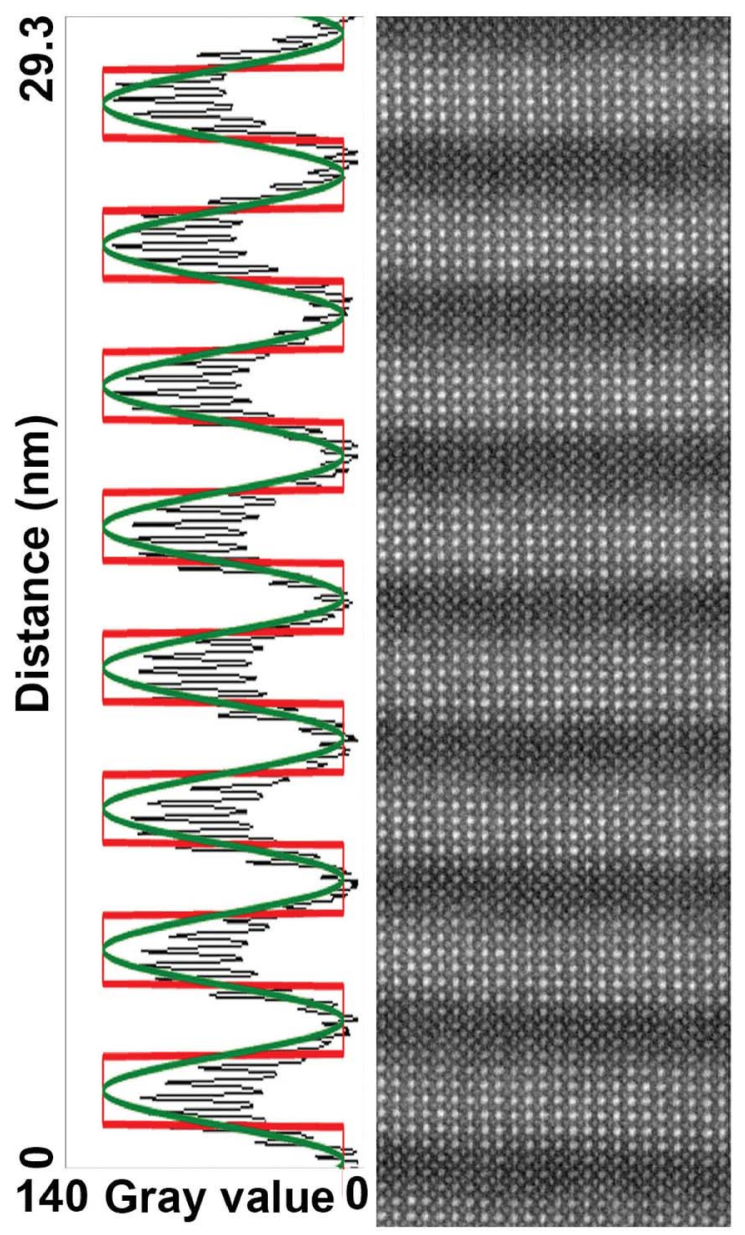

Fig. 8 HAADF-STEM image (right) and intensity profile (left) over the same region of the $\left[\left(\mathrm{YFeO}_{3}\right)_{5}\left(\mathrm{LaFeO}_{3}\right)_{5}\right]_{40}$ heterostructure. The red and green functions superimposed on the intensity profile correspond to the crenel and sine-wave modulation models used to produce the XRD simulations. The gradual overall decrease in peak height from top to bottom is due to varying cross-section thickness. 
$\left(\mathrm{LaFeO}_{3}\right)_{5}\left(\mathrm{YFeO}_{3}\right)_{5}$ superlattice was then constructed in which the A-site occupancy varied sinusoidally from a pure YO layer to a pure $\mathrm{LaO}$ in a period of five layers (Fig. S2 $\dagger$ ). Non-integer occupation of the A-sites was treated using a mean-field approach in which the cation potential is the occupancy weighted average of that for $\mathrm{Y}^{3+}$ and $\mathrm{La}^{3+}$. The polarization of the modulated structure was then calculated using static charges, with an overall polarization of $2.4 \mu \mathrm{C} \mathrm{cm}^{-2}$, only slightly less than that of the fully ordered superlattice. The symmetry arguments above show that the polarity does not depend on the nature (sine wave or crenel) of the modulation, only on its periodicity and composition.

Low temperature growth of orthorhombic $\mathrm{LnFeO}_{3}$ films affords domains with the $b^{+}$tilt both in- and out of plane. ${ }^{52-54}$ The relative orientation of the in-phase tilt to the A site ordering is critical in determining the presence of polarity - when the inphase tilt is aligned with the A site ordering, the symmetry is polar $P 2{ }_{1} m a$, whereas alignment of the out-of-phase tilt along this direction affords non-polar $P 2_{1} / m$ (Fig. 3d). In order to identify the relative orientation of the $b^{+}$tilt the pole figure of the (111) reflection of the Pnma orthoferrite subcell was measured. This reflection of the perovskite subcell allows for the differentiation of the $b^{+}$tilt orientation between polar $P 2{ }_{1} m a$ and non-polar $P 2_{1} / m$ (Fig. 9d) structures. The comparison between the measured pole figures (Fig. 9a) and the expected pole figures for untwinned crystals (Fig. 9b and c) show that both tilt orientations with respect to the cation ordering direction are present (confirmed by measuring reciprocal space maps around the (111) reflections, Fig. S9†), and thus both nonpolar $P 2_{1} / m$ and polar $P 2_{1} m a$ structures are formed in an approximate ratio of $5 / 1$ based on diffracted intensities. This would be expected to produce both polar and non-polar domains in the film, and is consistent with the small calculated energy differences between these two structures, which both grow although the polar one is not matched with the substrate tilt pattern.

\section{Magnetic properties}

All the $\mathrm{LnFeO}_{3}$ phases display weak ferromagnetism at all temperatures below $T_{\mathrm{N}}$, except $\mathrm{DyFeO}_{3}$ which is purely antiferromagnetic below $37 \mathrm{~K} .{ }^{55} \mathrm{LaFeO}_{3}$ and $\mathrm{YFeO}_{3}\left(T_{\mathrm{N}}=648 \mathrm{~K}\right.$ and
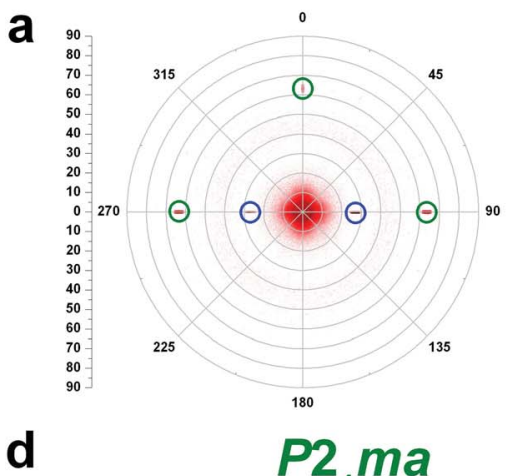

d

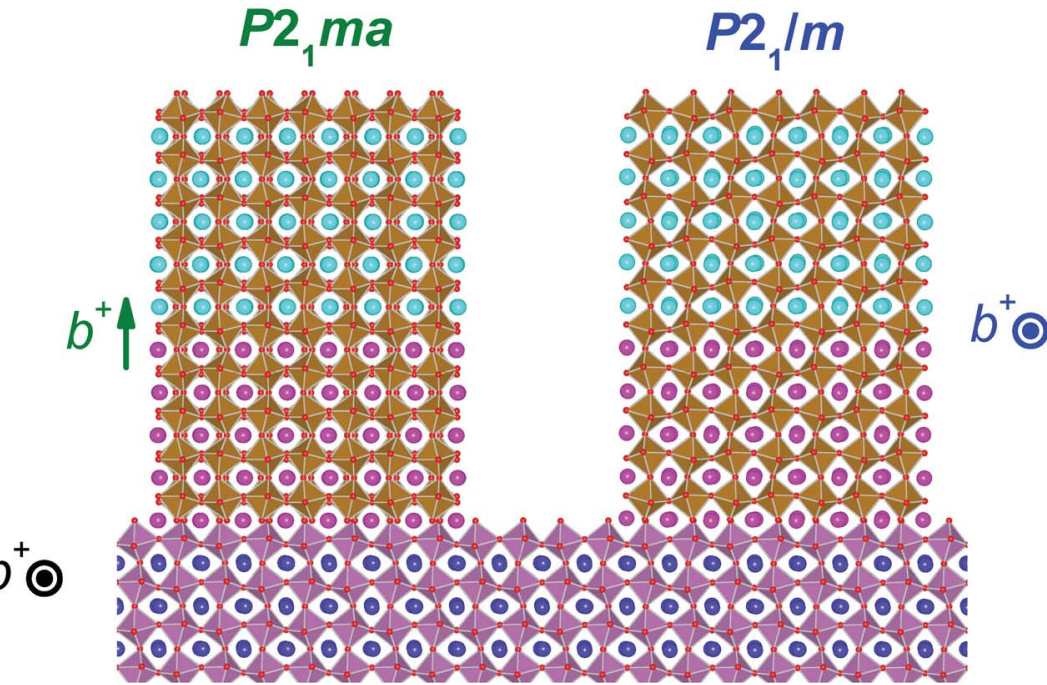

b

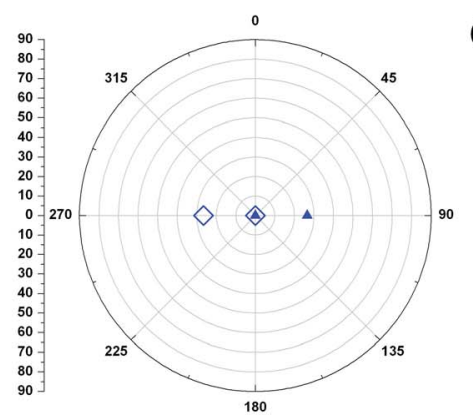

C
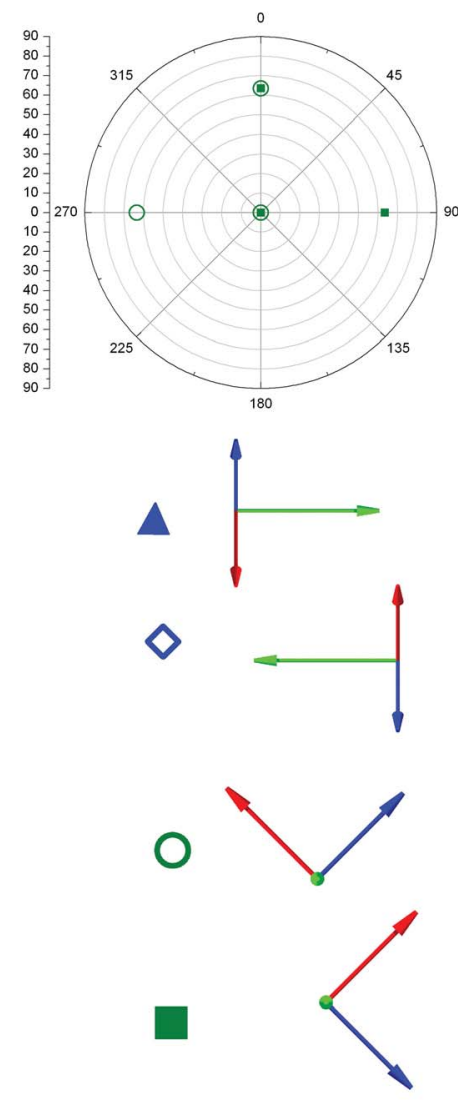

Fig. 9 (a) Measured pole figure of the (111) reflection of the Pnma subcell showing two different domains corresponding to the non polar phase (peaks highlighted as blue circles) and the polar phase (peaks highlighted as green circles). (b) Simulated pole figure for the non-polar $P 2_{1} / m$ phase considering two variants corresponding to different orientations of the $2 a_{p} b^{+}$axis. (c) Simulated pole figure for the polar $P 21 m a$ phase considering two variants corresponding to different orientations of the $\sqrt{ } 2 a_{p} a^{-}$axes. (d) Representation of the two phases obtained from the $A$ cation ordering stacked along the $b^{+}$tilt (left structure) and perpendicular to the $b^{+}$tilt (right structure) together with the substrate. The key on the bottom right explains the relationship between the Pnma perovskite subcell orientations in the two domains seen for each of the two phases (the $2 a_{p}$ direction is shown in green, the two $\sqrt{2} a_{p}$ directions in blue and red). 
$740 \mathrm{~K}$, respectively) both adopt the $\Gamma_{4}$ magnetic structure with the weak ferromagnetic moment along the $2 a_{\mathrm{p}} b^{+}$tilt Pnma axis. The alternative $\Gamma_{2}$ magnetic structure, where the canted moment lies along $c_{\text {Pnma }}=\sqrt{ } 2 a_{\mathrm{p}}$, has been observed in $\mathrm{YFeO}_{3}$ above 70 kOe. ${ }^{56}$ The sensitivity of the weak ferromagnetism in $\mathrm{LaFeO}_{3}$ to the out-of-phase tilts via their control of the antisymmetric exchange and single-ion anisotropy ${ }^{57}$ may produce deviations from the bulk magnetic structure in the heterostructure driven by interfacial and ferroelastic strains.

Absolute magnetization measurements on heterostructure grown on $\mathrm{DyScO}_{3}$ substrates using standard magnetometry were not possible as the $\mathrm{DyScO}_{3}$ paramagnetism dominates the film response. ${ }^{58}$ Magnetization was measured on a superlattice grown on $\mathrm{SrTiO}_{3}$ (Fig. S10†). The remanent in-plane magnetization is $0.011 \pm 0.001 \mu_{\mathrm{B}}$ per formula unit which is similar to the reported value for $\mathrm{YFeO}_{3}$ films. ${ }^{52}$ The in- and out-of-plane antiferromagnetic susceptibilities of the heterostructure were determined to be $(11.3 \pm 0.3) \times 10^{-7}$ and $(5.4 \pm 0.6) \times 10^{-7} \mu_{\mathrm{B}} /$ Oe, respectively. These are of the same order found in $\mathrm{YFeO}_{3}$ single crystals at room temperature,$^{55}$ demonstrating that the antiferromagnetic anisotropy strength in the heterostructure is comparable to the bulk properties of its components and thus that the heterostructure is magnetically ordered at room temperature.

A qualitative characterization of the magnetic hysteresis loops has been performed using the magneto-optic measurements on a film grown on DSO substrates. Fig. 10a shows the MOKE loops of a $\left(\mathrm{YFeO}_{3}\right)_{5} /\left(\mathrm{LaFeO}_{3}\right)_{5}$ heterostructure with the magnetic field parallel to several high symmetry directions (Fig. 10d presents the relative orientation of substrate and films). We measured sharp hysteresis loops, similar to those observed on $\mathrm{LaFeO}_{3}$ thin films of similar thickness grown under the same conditions (Fig. 10c). The analysis of the magnetic anisotropy of these films and the absence of weak ferromagnetism when the magnetic field is applied parallel to the $[010]_{\mathrm{pc}}$ axis confirms the $\Gamma_{4}$ magnetic configuration of the heterostructure films. The observed negative slope of the hysteresis loop for the heterostructure and $\mathrm{LaFeO}_{3}$ is due to the contribution of domains with the $b_{P n m a}$ axis out of plane (eqn (S1) and $(\mathrm{S} 2) \dagger$ ) confirming the twinning (and the presence of polar and non-polar domains) described previously. The degree of the negative slope is likely to be proportional to the number of structural domains grown with the $b_{P n m a}$-axis out-of-plane.
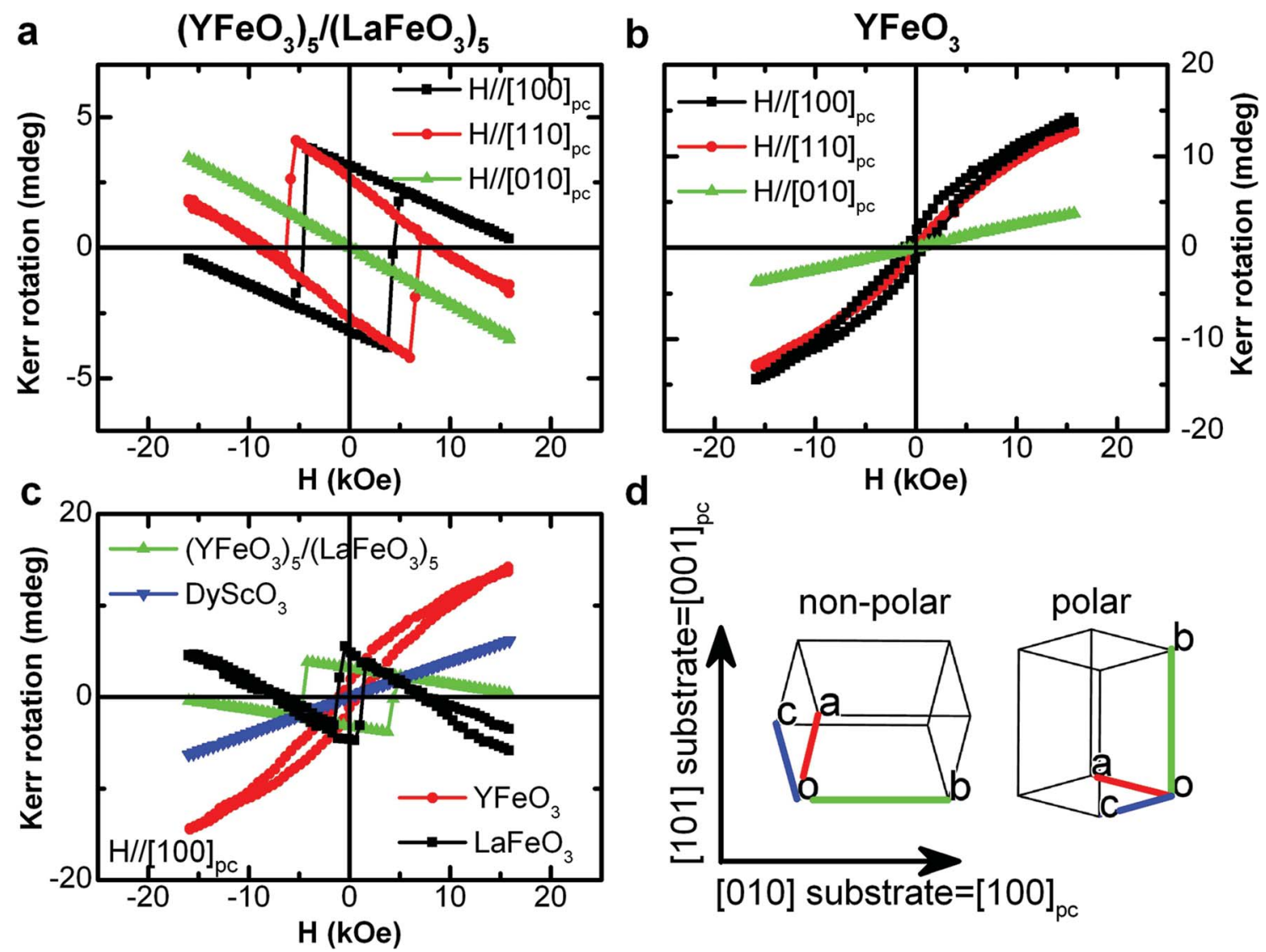

Fig. 10 Magnetic hysteresis loops measured by longitudinal MOKE at room temperature on (a) the $\left(\mathrm{YFeO}_{3}\right)_{5} /\left(\mathrm{LaFeO}_{3}\right)_{5}$ heterostructure and (b) the thin $\mathrm{YFeO}_{3}$ film both grown on $\mathrm{DyScO}_{3}(101)_{P_{n m a}}$ substrates, with magnetic field applied in different directions with respect to DyScO 3 $[010]_{P n m a}$ or $[100]_{p c}$ in orthorhombic and pseudo-cubic notations, respectively. (c) Comparison of MOKE hysteresis loops measured on blank substrate $\mathrm{DySCO}_{3}(101)_{P n m a}$ and on thin films of $\left(\mathrm{YFeO}_{3}\right)_{5} /\left(\mathrm{LaFeO}_{3}\right)_{5}, \mathrm{YFeO}_{3}$ and $\mathrm{LaFeO}_{3}$ grown on DyScO${ }_{3}(101)_{P_{n m a}}$ substrates, respectively. The magnetic field is applied along the $\mathrm{DySCO}_{3}[010]_{\text {Pnma }}$ axis. (d) Three-dimensional drawings of orthorhombic unit cells corresponding to the polar and non-polar phases in $\left(\mathrm{YFeO}_{3}\right)_{5} /\left(\mathrm{LaFeO}_{3}\right)_{5}$ and their orientations with respect to orthorhombic (Pnma) or pseudo-cubic crystal axes of DyScO 3 $(101)_{\text {Pnma }}$ substrate. 


\section{Non-linear optical properties}

To confirm the broken spatial inversion symmetry and to verify the polar point group of the $5: 5$ heterostructure we performed measurements of the optical second harmonic generation (SHG) polar plots on $\left[\left(\mathrm{YFeO}_{3}\right)_{5}\left(\mathrm{LaFeO}_{3}\right)_{5}\right]_{40}$ heterostructure and on a blank $\mathrm{DyScO}_{3}$ substrate, respectively. SHG provides a necessary condition for polar dipolar order. ${ }^{59}$ Fig. 11 shows the dependence of the SHG $I^{2 \omega}(\theta)$ intensity $v s$. polarizer rotational angle. Numerical analysis using eqn $(\mathrm{S} 3) \dagger$ agrees with the point symmetry group $2 \mathrm{~mm}$, of the $P 2{ }_{1} \mathrm{ma}$ space group of the polar phase. Measurements on a blank $\mathrm{DyScO}_{3}$ substrate yielded an 8 times smaller absolute SHG response and agreed with the nonpolar group $\mathrm{mmm}$ expected for Pnma, confirming the polar nature of the heterostructure, similar to the data reported for strained $\mathrm{EuTiO}_{3} \cdot{ }^{21}$

\section{Piezoelectric force microscopy}

The functional nature of the structurally generated polarization was demonstrated by measuring the piezoresponse between interdigitated electrodes with the cantilever parallel to the electrodes (ESI Fig. S13 $\dagger$ ). The lateral and vertical displacement amplitudes are plotted in Fig. 12a and b with the negative sign used to indicate $180^{\circ}$ phase difference between the applied voltage and measured cantilever displacement. Changing the voltage offset used alters both the displacement amplitudes and signs, as expected from the electric field dependence of the piezoelectric coefficient. ${ }^{60}$ In order to verify the piezoelectric nature of the response and the electric polarization direction, finite element simulations were performed for the measured sample geometry using the relaxed-ion piezoelectric tensor with a calculated $\mathrm{d}_{33}$ of $25.88 \mathrm{pC} \mathrm{N}^{-1}$ for the $\left(\mathrm{LaFeO}_{3}\right)_{1}\left(\mathrm{YFeO}_{3}\right)_{1}$ superlattice. The electrical polarization lies in the sample plane, perpendicular to the cation ordering direction as expected from the $P 2_{1} m a$ symmetry produced orientation of the $b^{+}$tilt. $d_{33}$ for the heterostructures was estimated within an order of magnitude as $10 \mathrm{pC} \mathrm{N}^{-1}$. The agreement between this estimate and

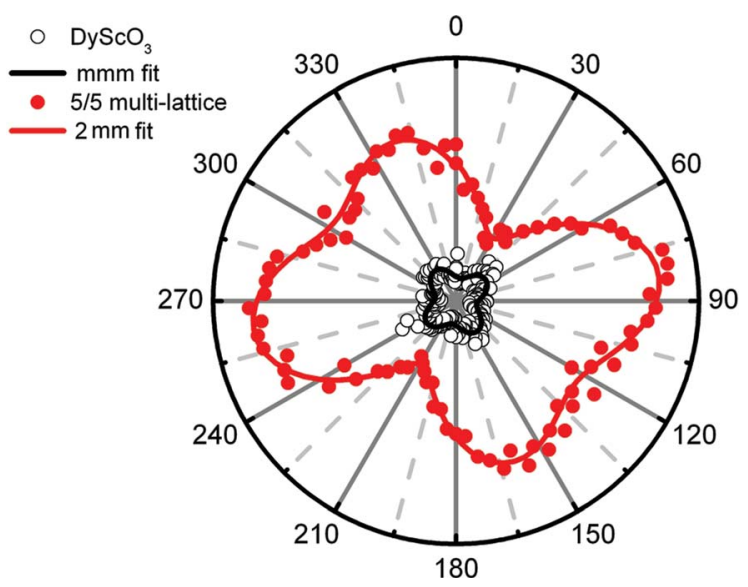

Fig. 11 Optical second harmonic response vs. rotational angle of the polarizer. Filled (open) symbols and red (black) solid line represent data points and the corresponding fit for the film and blank $\mathrm{DySCO}_{3}(101)_{P n m a}$ substrate with point symmetry group $2 \mathrm{~mm}$ and $\mathrm{mmm}$, respectively.
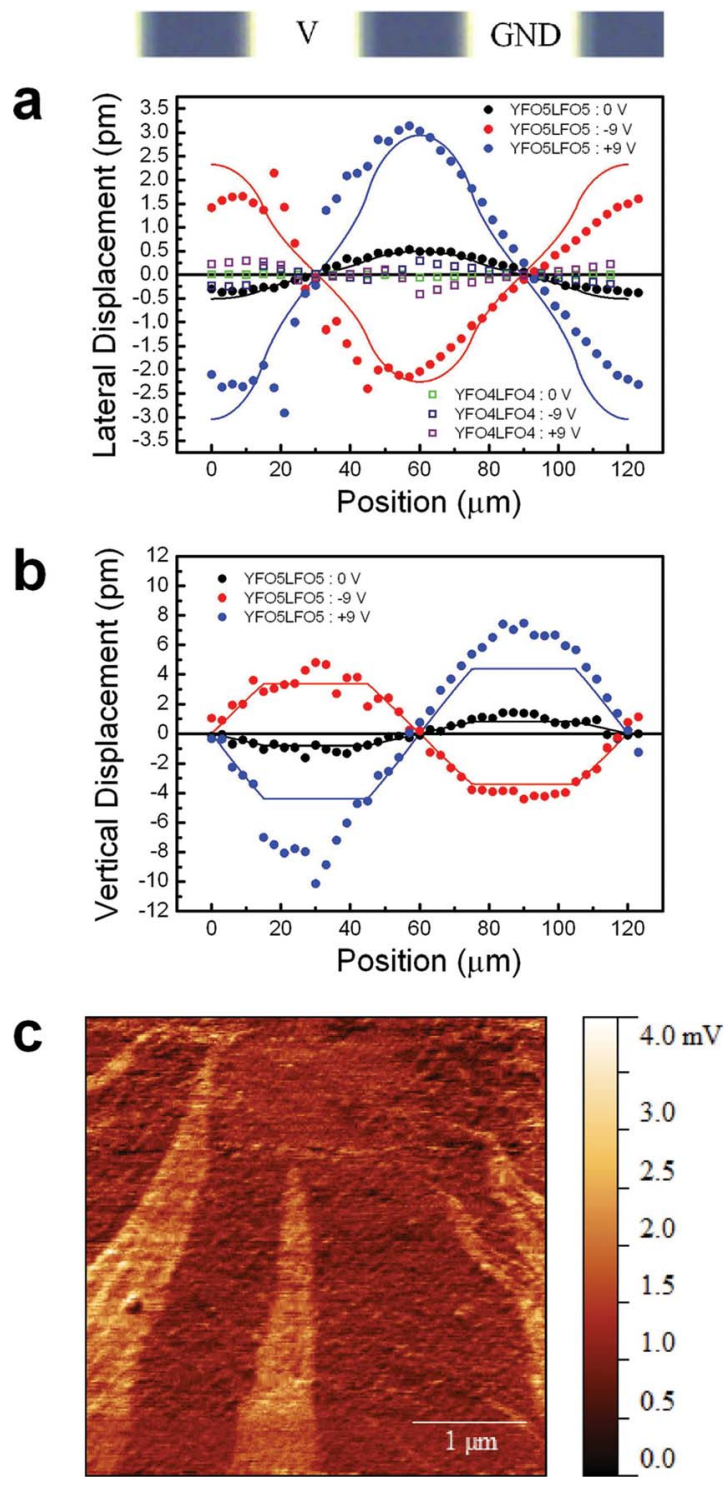

Fig. 12 (a) Lateral and (b) vertical piezoresponse profile measurements (solid circles) and simulations (lines) for the $\left(\mathrm{LaFeO}_{3}\right)_{5}\left(\mathrm{YFeO}_{3}\right)_{5}$ and $\left(\mathrm{LaFeO}_{3}\right)_{4}\left(\mathrm{YFeO}_{3}\right)_{4}$ (square) thin films across a series of interdigitated electrodes shown in the top optical micrograph. The measurements and simulations are shown for three values of voltage offset, $0 \mathrm{~V}$ and $\pm 9 \mathrm{~V}$. (c) Scanning-mode PFM image of the $\left(\mathrm{LaFeO}_{3}\right)_{5}\left(\mathrm{YFeO}_{3}\right)_{5}$ film measured at a cantilever resonance using $1 \mathrm{~V}$ amplitude at $320 \mathrm{kHz}$. The vertical response amplitude is plotted, bright areas indicating polar domains whilst the dark areas are non-polar regions.

simulation confirms the piezoelectric origin of the sample surface displacements (Fig. 12a). Measurements with the cantilever perpendicular to the electrodes give a lateral signal within the noise level, indicating negligible polarization in this direction. We performed control experiments on separate $\left[\left(\mathrm{YFeO}_{3}\right)_{4}\left(\mathrm{LaFeO}_{3}\right)_{4}\right]_{50}, \quad \mathrm{YFeO}_{3}$ and $\mathrm{LaFeO}_{3}$ films grown on $\mathrm{DyScO}_{3}$ under identical conditions to those used to grow the heterostructures and did not measure any detectable piezoelectric effect (Fig. 12a and S14 $\dagger$ ). Scanning-mode PFM imaging of the surface of the $\left[\left(\mathrm{YFeO}_{3}\right)_{5}\left(\mathrm{LaFeO}_{3}\right)_{5}\right]_{40}$ heterostructure reveals both polar (bright area) and non-polar (darker areas) regions (Fig. 12c) with the polar region covering $25 \%$ of the 
surface. The sample surface is very smooth with a root mean square roughness of $1 \mathrm{~nm}$ and no correlation was observed between the topography and piezoresponse signals.

\section{Conclusions}

$\mathrm{LaFeO}_{3}$ and $\mathrm{YFeO}_{3}$ are isosymmetric, non-polar and magnetically ordered at room temperature - spatial inversion symmetry is present, but time reversal symmetry is broken. Growth of alternating blocks of odd numbers of perovskite unit cells orders the A site $\mathrm{La}^{3+}$ and $\mathrm{Y}^{3+}$ cations. The cation order along the in-phase octahedral tilt removes the inversion centre in the component. The resulting uncompensated cation displacements from the centroids of their oxygen coordination polyhedra in the plane of the cation order produce a polarization perpendicular to the growth direction. The correct coupling of the translational (A site order) and point (octahedral in-phase rotation) symmetry is essential - if this is not achieved, nonpolar structures result as shown by the presence of polar and non-polar regions in the PFM measurements. Rather than relying on a sharp compositional variation at the interface to break the symmetry, a more general approach is devised where coupled A cation ordering and specific mode distortions are the driving mechanism to create a polar system. This demonstrates the robustness of this approach to imperfection at the interfaces since a non-square-wave modulation of the composition will not affect the symmetry as long as the periodicity of the heterostructure remains correct. The A site occupancy modulation does not need to be square-wave to produce the polarization; it is the correspondence of the periodicity to an odd number of blocks of each component which is essential. The magnetic order of the component $\mathrm{AFeO}_{3}$ blocks is retained in the heterostructure, with the structural polarity and functional piezoresponse imposed by the growth. The combination of magnetism and polarization at room temperature is thus achieved through the growth-controlled loss of symmetry. This mechanism does not require restrictive chemical criteria in the component ions $\left(\mathrm{d}^{0}\right.$ or $\mathrm{s}^{2}$ configurations are not a pre-requisite, for example) and can be tuned via block size and chemical composition while still requiring only two distinct isosymmetric starting units, offering considerable potential diversity in property combination and control.

\section{Acknowledgements}

This work is funded by the European Research Council (ERC Grant agreement 227987 RLUCIM). EDM, NES and NAI also acknowledge RFBR Grant \#13-02-12450. Work done at WVU by PB and DL was supported by a Research Challenge Grant from the West Virginia Higher Education Policy Commission (HEPC.dsr.12.29). MJR thanks the Royal Society for the award of a Research Professorship.

\section{Notes and references}

1 N. A. Hill, J. Phys. Chem. B, 2000, 104, 6694-6709.

2 J. van Suchtelen, Philips Res. Rep., 1972, 27, 28-37.
3 Y. Tokura, J. Magn. Magn. Mater., 2007, 310, 1145.

4 Y. Tokura and S. Seki, Adv. Mater., 2010, 22, 1554-1565.

5 N. J. Perks, R. D. Johnson, C. Martin, L. C. Chapon and P. G. Radaelli, Nat. Commun., 2012, 3, 1277.

6 M. Uehara, S. Mori, C. H. Chen and S.-W. Cheong, Nature, 1999, 399, 560-563.

7 L. Bellaiche and J. Iniguez, Phys. Rev. B: Condens. Matter Mater. Phys., 2013, 88, 014104.

8 A. A. Taskin, A. N. Lavrov and Y. Ando, Appl. Phys. Lett., 2005, 86, 091910.

9 E. Bousquet, M. Dawber, N. Stucki, C. Lichtensteiger, P. Hermet, S. Gariglio, J.-M. Triscone and P. Ghosez, Nature, 2008, 452, 732-736.

10 K. Ueda, H. Tabata and T. Kawai, Science, 1998, 280, 10641066.

11 N. A. Benedek and C. J. Fennie, Phys. Rev. Lett., 2011, 106, 107204.

12 N. A. Benedek, D. A. Mulder and C. J. Fennie, J. Solid State Chem., 2012, 195, 11-20.

13 A. T. Mulder, N. A. Benedek, J. M. Rondinelli and C. J. Fennie, Adv. Funct. Mater., 2013, 23, 4810-4820.

14 J. M. Rondinelli and C. J. Fennie, Adv. Mater., 2012, 24, 19611968.

15 K. Kishida, K. Goto and H. Inui, Acta Crystallogr., Sect. B: Struct. Sci., 2009, 65, 405-415.

16 T. Fukushima, A. Stroppa, S. Picozzi and J. M. Perez-Mato, Phys. Chem. Chem. Phys., 2011, 13, 12186-12190.

17 G. Rogez, N. Viart and M. Drillon, Angew. Chem., Int. Ed., 2010, 49, 1921-1923.

18 A. Stroppa, P. Barone, P. Jain, J. M. Perez-Mato and S. Picozzi, Adv. Mater., 2013, 25, 2284-2290.

19 H. Y. Hwang, Y. Iwasa, M. Kawasaki, B. Keimer, N. Nagaosa and Y. Tokura, Nat. Mater., 2012, 11, 103-113.

20 K. Rogdakis, J. W. Seo, Z. Viskadourakis, Y. Wang, L. F. N. Ah Qune, E. Choi, J. D. Burton, E. Y. Tsymbal, J. Lee and C. Panagopoulos, Nat. Commun., 2012, 3, 1064.

21 J. H. Lee, L. Fang, E. Vlahos, X. Ke, Y. W. Jung, L. F. Kourkoutis, J.-W. Kim, P. J. Ryan, T. Heeg, M. Roeckerath, V. Goian, M. Bernhagen, R. Uecker, P. C. Hammel, K. M. Rabe, S. Kamba, J. Schubert, J. W. Freeland, D. A. Muller, C. J. Fennie, P. Schiffer, V. Gopalan, E. Johnston-Halperin and D. G. Schlom, Nature, 2010, 466, 954-958.

22 Y. Ogawa, H. Yamada, T. Ogasawara, T. Arima, H. Okamoto, M. Kawasaki and Y. Tokura, Phys. Rev. Lett., 2003, 90, 217403-2174034.

23 Z. Zanolli, J. C. Wojdel, J. Iniguez and P. Ghosez, Phys. Rev. B: Condens. Matter Mater. Phys., 2013, 88, 060102.

24 J. E. Kleibeuker, G. Koster, W. Siemons, S. Dubbink, B. Kuiper, J. L. Blok, C.-H. Yang, J. Ravichandran, R. Ramesh, J. E. T. Elshof, D. H. A. Blank and G. Rijnders, Adv. Funct. Mater., 2010, 20, 3490-3496.

25 G. Koster, B. L. Kropman, G. Rijnders, D. H. A. Blank and H. Rogalla, Appl. Phys. Lett., 1998, 73, 2920.

26 S. Kalinin and A. Gruverman, Scanning Probe Microscopy: Electrical and Electromechanical Phenomena at the Nanoscale, Springer, 2007. 
27 K. Momma and F. Izumi, J. Appl. Crystallogr., 2008, 41, 653658.

28 H. T. Stokes, D. M. Hatch and B. J. Campbell, in stokes.byu.edu/isotropy.html, 2007.

29 J. P. Perdew, K. Burke and M. Ernzerhof, Phys. Rev. Lett., 1996, 77, 3865-3868.

30 S. L. Dudarev, G. A. Botton, S. Y. Savrasov, C. J. Humphreys and A. P. Sutton, Phys. Rev. B: Condens. Matter Mater. Phys., 1998, 57, 1505-1509.

31 G. Kresse and J. Furthmüller, Phys. Rev. B: Condens. Matter Mater. Phys., 1996, 54, 11169-11186.

32 G. Kresse and D. Joubert, Phys. Rev. B: Condens. Matter Mater. Phys., 1999, 59, 1758-1775.

33 M. Gajdoš, K. Hummer, G. Kresse, J. Furthmüller and F. Bechstedt, Phys. Rev. B: Condens. Matter Mater. Phys., 2006, 73, 045112.

34 P. Giannozzi, S. Baroni, N. Bonini, M. Calandra, R. Car, C. Cavazzoni, D. Ceresoli, G. L. Chiarotti, M. Cococcioni, I. Dabo, A. Dal Corso, S. de Gironcoli, S. Fabris, G. Fratesi, R. Gebauer, U. Gerstmann, C. Gougoussis, A. Kokalj, M. Lazzeri, L. Martin-Samos, N. Marzari, F. Mauri, R. Mazzarello, S. Paolini, A. Pasquarello, L. Paulatto, C. Sbraccia, S. Scandolo, G. Sclauzero, A. P. Seitsonen, A. Smogunov, P. Umari and R. M. Wentzcovitch, J. Phys.: Condens. Matter, 2009, 21, 395502.

35 N. Spaldin, J. Solid State Chem., 2012, 195, 2-10.

36 J. D. Gale and A. L. Rohl, Mol. Simul., 2003, 29, 291-341.

37 B. G. J. Dick and A. W. Overhauser, Phys. Rev., 1958, 112, 90103.

38 R. A. Buckingham, Proc. R. Soc. London, Ser. A, 1938, 168, 264-283.

39 M. S. Dyer, C. Collins, D. Hodgeman, P. A. Chater, A. Demont, S. Romani, R. Sayers, M. F. Thomas, J. B. Claridge, G. R. Darling and M. J. Rosseinsky, Science, 2013, 340, 847-852.

40 M. S. D. Read, M. S. Islam, G. W. Watson, F. King and F. E. Hancock, J. Mater. Chem., 2000, 10, 2298-2305.

41 A. M. Glazer, Acta Crystallogr., Sect. B: Struct. Crystallogr. Cryst. Chem., 1972, 28, 3384-3392.

42 A. P. Levanyuk and D. G. Sannikov, Sov. Phys. - Usp., 1974, 17, 199-214.
43 M. R. Dolgos, U. Adem, A. Manjon-Sanz, X. Wan, T. P. Comyn, T. Stevenson, J. Bennett, A. J. Bell, T. T. Tran, P. S. Halasyamani, J. B. Claridge and M. J. Rosseinsky, Angew. Chem., Int. Ed., 2012, 51, 10770-10775.

44 S. van Smaalen, Zeitschrift für Kristallographie, 2004, 219, 681-691.

45 J. M. Perez-Mato, D. Orobengoa and M. I. Aroyo, Acta Crystallogr., Sect. A: Cryst. Phys., Diffr., Theor. Gen. Crystallogr., 2010, 66, 558-590.

46 J. M. Perez-Mato, D. Orobengoa, M. I. Aroyo and L. Elcoro, J. Phys.: Conf. Ser., 2010, 226, 012011.

47 E. S. Tasci, G. de la Flor, D. Orobengoa, C. Capillas, J. M. Perez-Mato and M. I. Aroyo, EPJ Web Conf., 2012, 22, 00009.

48 I. Orlov, L. Palatinus and G. Chapuis, Angew. Chem., Int. Ed., 2008, 41, 1182-1186.

49 I. Orlov, N. Schoeni and G. Chapuis, http://it.iucr.org/ resources/finder/.

50 F. Boucher, M. Evain and V. Petricek, Acta Crystallogr., Sect. B: Struct. Crystallogr. Cryst. Chem., 1996, 52, 100-109.

51 R. L. White, J. Appl. Phys., 1969, 40, 1061-1069.

52 J. Scola, P. Boullay, W. Noun, E. Popova, Y. Dumont, A. Fouchet and N. Keller, J. Appl. Phys., 2011, 110, 043928.

53 L. Bi, H.-S. Kim, G. F. Dionne, C. A. Ross, H. Paik and Y. C. Park, Appl. Phys. Lett., 2009, 95, 121908.

54 P. Boullay, A. David, W. C. Sheets, U. Lüders, W. Prellier, H. Tan, J. Verbeeck, G. Van Tendeloo, C. Gatel, G. Vincze and Z. Radi, Phys. Rev. B: Condens. Matter Mater. Phys., 2011, 83, 125403.

55 D. Treves, Phys. Rev., 1962, 125, 1843-1853.

56 I. S. Jacobs, H. F. Burne and L. M. Levinson, J. Appl. Phys., 1971, 42, 1631.

57 C. Weingart, N. Spaldin and E. Bousquet, Phys. Rev. B: Condens. Matter Mater. Phys., 2012, 86, 094413.

58 X. Ke, C. Adamo, D. G. Schlom, M. Bernhagen, R. Uecker and P. Schiffer, Appl. Phys. Lett., 2009, 94, 152503.

59 N. Bloembergen, Non linear optics, W. A. Benjamin Inc., New York, 1965.

60 D. Damjanovic, Rep. Prog. Phys., 1996, 61, 1267-1324. 